\title{
TAYLOR'S FORMULA FOR MAPS: THE REVERSE PROBLEM
}

\author{
ARTHUR SARD
}

1. Introduction. Suppose that $X, Y$ are Banach spaces and that $\Omega \subset X$ is an open set such that the line segment $[0, x] \subset \Omega$ whenever $x \in \Omega$. Denote by $L_{i}(X, Y)$ the space of multilinear continuous operators on $X \times \ldots \times X$ ( $i$ factors) to $Y$, with the Banach norm, and by $S_{i}(X, Y)$ the subspace of symmetric operators.

If $f: \Omega \rightarrow Y$ is a $C^{p}$-map, $p \geqq 1$, then $f$ and its derivatives satisfy the Taylor formulas:

(1) $f^{k}(x)=a_{k}+\ldots+\frac{a_{p-1}}{(p-k-1) !} x^{p-k-1}+\int_{0}^{1} \frac{(1-t)^{p-k-1}}{(p-k-1) !} \varphi(t x) x^{p-k} d t$,

where

$$
k=0, \ldots, p-1 ; x \in \Omega,
$$

$$
\varphi=f^{p}: \Omega \rightarrow S_{p}(X, Y)
$$

is the $p$-th derivative of $f$, and

$[1,2,3]$.

$$
a_{i}=f^{i}(0) \in S_{i}(X, Y), \quad i=0, \ldots, p-1
$$

Now consider the reverse problem. Suppose that we are given a $C^{0}$-map of $\Omega$ into the symmetric continuous multilinear operators of order $p$

$$
\psi: \Omega \rightarrow S_{p}(X, Y)
$$

and symmetric continuous multilinear operators

$$
b_{i} \in S_{i}(X, Y), \quad i=0, \ldots, p-1 \text {; }
$$

and suppose that we define the map $g: \Omega \rightarrow Y$ by the relations

$$
\begin{gathered}
g(x)=b_{0}+\ldots+\frac{b_{p-1}}{(p-1) !} x^{p-1}+r(x), \\
r(x)=\int_{0}^{1} \frac{(1-t)^{p-1}}{(p-1) !} \psi(t x) x^{p} d t, \quad x \in \Omega .
\end{gathered}
$$

What can we say about the derivatives of $g$ and $r$ ? 
Proposition 1 below implies that $r^{p}=\psi$ if and only if $\psi$ is the $p$-th derivative of a map. Proposition 3 implies that, if $\psi \in C^{1}$, then $\psi$ is the $p$-th derivative of a map if and only if the first derivative $\psi^{1}$, which is a map into $S_{1}\left(X, S_{p}(X, Y)\right)$, is symmetric in its $p+1$ arguments, that is, if and only if $\psi^{1}(x) \in S_{p+1}(X, Y)$, $x \in \Omega$.

It is worthwile, I think, to study the behaviour of $g$ and $r$ in the case in which $\psi$ is not a $p$-th derivative. For example, $\psi$ may be known to be an approximation of the $p$-th derivative of the unknown map $f: \Omega \rightarrow Y$. We may take $g$ as an approximation of $f$, based on $\psi$ and the approximate initial values $b_{0}, \ldots, b_{p-1}$. We would then be interested in the extent to which the derivatives $g^{k}$ approximate $f^{k}, k \leqq p$. Formulas (22), (23), and (19) below give the errors $g^{k}-f^{k}$ in terms of $b_{i}-a_{i}, i<p$, and $\psi^{j}-\varphi^{j}, j \leqq k$, in the case in which $\psi, \varphi \in C^{k}$. These errors are appraised in terms of the norms of $b_{i}-a_{i}$ and $\psi^{j}-\varphi^{j}$ in Section 7.

Formulas (16), (10) give the derivatives $r^{k}, k \leqq p$, in terms of $\psi, \psi^{1}, \ldots, \psi^{k}$.

2. The case in which $\psi$ is a $p$-th derivative.

Proposition 1. If $\psi$ is the $p$-th derivative of a map on $\Omega$ to $Y$, then $r \in C^{p}$, $g \in C^{p}$, and

$$
\left\{\begin{array}{l}
r^{p}=g^{p}=\psi \\
r^{k}(x)=\int_{0}^{1} \frac{(1-t)^{p-k-1}}{(p-k-1) !} \psi(t x) x^{p-k} d t, \quad k<p, \quad x \in \Omega .
\end{array}\right.
$$

Conversely, if $g$ or $r$ is in $C^{p}$, then the other is also, and

$$
\begin{cases}r^{p}=g^{p}, & r^{p}(0)=\psi(0), \\ r^{k}(0)=0, & g^{k}(0)=b_{k}, \quad k<p ;\end{cases}
$$

however $r^{p}$ need not equal $\psi$.

Proof. Say that $\psi=f^{p}$. Then $f \in C^{p}$, since $\psi \in C^{0}$. By (1) and (4),

Hence

$$
r(x)=f(x)-a_{0}-\ldots-\frac{a_{p-1}}{(p-1) !} x^{p-1}, \quad x \in \Omega .
$$

and

$$
g(x)=f(x)+b_{0}-a_{0}+\ldots+\frac{b_{p-1}-a_{p-1}}{(p-1) !} x^{p-1},
$$

Furthermore,

$$
g^{p}(x)=f^{p}(x)=\psi(x)=r^{p}(x) .
$$

$r^{k}(x)=f^{k}(x)-a_{k}-\ldots-\frac{a_{p-1}}{(p-k-1) !} x^{p-k-1}=\int_{0}^{1} \frac{(1-t)^{p-k-1}}{(p-k-1) !} \psi(t x) x^{p-k} d t, \quad k<p$,

by (1).

Conversely, if $g$ or $r \in C^{p}$, then so is the other and $g^{p}=r^{p}$, by (3). 
By (4),

$$
\|r(x)\| \leqq \frac{\|\psi, x\|\|x\|^{p}}{p !}, \quad x \in \Omega,
$$

where, in a special notation,

$$
\|\psi, x\|=\sup _{t \in[0,1]}\|\psi(t x)\|<\infty,
$$

because the segment $[0, x]$ is compact. On the other hand,

$$
r(x)=r(0)+\ldots+\frac{r^{p-1}(0)}{(p-1) !} x^{p-1}+\int_{0}^{1} \frac{(1-t)^{p-1}}{(p-1) !} r^{p}(t x) x^{p} d t .
$$

This with (7) implies that

and

$$
r^{k}(0)=0, \quad k<p
$$

$$
g^{k}(0)=b_{k}+r^{k}(0)=b_{k} \text {. }
$$

Furthermore (4) implies that

$$
r(x)-\psi(0) x^{p} / p !=\theta(x) x^{p}, \quad x \in \Omega,
$$

where $\theta(x) \rightarrow 0$ as $x \rightarrow 0$. By (8), a similar relation holds with $\psi(0)$ replaced by $r^{p}(0)$. Hence $r^{p}(0)=\psi(0)$, and (6) is established.

That $r^{p}$ need not equal $\psi$ is to be expected, since the differential of $x$ need not be collinear with the segment $[0, x]$. It is sufficient to cite one instance. Take $X=R^{2}=\Omega, Y=R, p=1$, and $\psi$ the map of $R^{2}$ into $S_{1}\left(R^{2}, R\right)=L_{1}\left(R^{2}, R\right)$ for which

Then

$$
\psi(u) x=u_{1} x_{2}, \quad u=\left(u_{1}, u_{2}\right) \in R^{2}, \quad x=\left(x_{1}, x_{2}\right) \in R^{2} .
$$

and

$$
r(x)=\int_{0}^{1} t x_{1} x_{2} d t=x_{1} x_{2} / 2
$$

$$
r^{1}(x) h=\left(h_{1} x_{2}+x_{1} h_{2}\right) / 2, \quad h=d x=\left(h_{1}, h_{2}\right) \in R^{2} .
$$

On the other hand

$$
\psi(x) h=x_{1} h_{2} .
$$

Thus $r^{1}(x) \neq \psi(x)$, unless $x=0$.

3. The derivatives of $\psi$. Since $\psi$ is a map into $S_{p}(X, Y)$, the value $\psi^{j}(x)$ of the $j$-th derivative, if existent, is an element of $S_{j}\left(X, S_{p}(X, Y)\right)$ and so may be identified with an element of $L_{j+p}(X, Y)$. It need not be true, however, that $\psi^{j}(x) \in S_{j+p}(X, Y)$.

Proposition 2. If for some $x \in \Omega$, the first derivative $\psi^{1}(x)$ exists and is symmetric in its $p+1$ arguments, then all subsequent derivatives which exist at $x$ are symmetric in all their arguments. 
Proof. By hypothesis, $h_{1}, x_{1}, \ldots, x_{p} \in X$ may be permuted arbitrarily in

$$
\psi^{1}(x) h_{1} x_{1} \ldots x_{p} \in Y \text {. }
$$

If $\psi^{2}(x)$ exists, it follows that $h_{1}, x_{1}, \ldots, x_{p}$ may be permuted arbitrarily in

$$
\psi^{2}(x) h_{2}\left(h_{1} x_{1} \ldots x_{p}\right) \in Y, \quad h_{2}=d x \in X .
$$

But this element equals

$$
\psi^{2}(x) h_{1} h_{2} x_{1} \ldots x_{p},
$$

since a derivative is symmetric in its differentials. Thus $h_{1}$ and therefore $h_{2}, x_{1}, \ldots, x_{p}$ may be permuted arbitrarily.

Iterating, we see that if $\psi^{j}(x)$ exists, then

$$
\psi^{j}(x) h_{1} \ldots h_{j} x_{1} \ldots x_{p}, h_{1}, \ldots, h_{j}, x_{1}, \ldots, x_{p} \in X,
$$

is invariant under permutations of the $j+p$ arguments.

Proposition 3. Suppose that $\psi \in C^{1}$. Then a necessary and sufficient condition that $\psi=r^{p}$ is that the first derivative $\psi^{1}$ be symmetric in its $p+1$ arguments.

Proof. Necessity is immediate: If $\psi=r^{p}$, then $\psi^{1}=r^{p+1}$, being a $(p+1)$-th derivative, is symmetric in its $p+1$ arguments.

Sufficiency. By Leibnitz' rule, which is valid because the segment $[0, x]$ is compact,

$$
r^{1}(x)=\int_{0}^{1} \frac{(1-t)^{p-1}}{(p-1) !} D_{x}\left[\psi(t x) x^{p}\right] d t, \quad x \in \Omega .
$$

In evaluating the interior derivative, we shall work with differentials, since the position of differentials of $x$ is critical and may not be changed arbitrarily. Note however that $p, t$, and $d t$ are scalars and commute with all other factors. Now

$$
\begin{gathered}
d_{x}\left[\psi(t x) x^{p}\right]=\psi(t x) d_{x} x^{p}+\left[d_{x} \psi(t x)\right] x^{p} \\
=p \psi(t x) x^{p-1} h+t \psi^{1}(t x) h x^{p}, \quad h=d x \in X, \quad x \in \Omega, \quad 0 \leqq t \leqq 1 .
\end{gathered}
$$

Our present hypothesis implies that

Hence

$$
\psi^{1}(t x) h x^{p}=\psi^{1}(t x) x^{p} h
$$

and

$$
D_{x}\left[\psi(t x) x^{p}\right]=p \psi(t x) x^{p-1}+t \psi^{1}(t x) x^{p}
$$

$$
r^{1}(x)=\int_{0}^{1} \frac{(1-t)^{p-1}}{(p-1) !}\left[p \psi(t x) x^{p-1}+t \psi^{1}(t x) x^{p}\right] d t, \quad x \in \Omega .
$$

Transforming the second term by parts, with $d_{t} \psi(t x)=\psi^{1}(t x) x d t$, we see that

$$
r^{1}(x)= \begin{cases}\psi(x), & \text { if } \quad p=1, \\ \int_{0}^{1} \frac{(1-t)^{p-2}}{(p-2) !} \psi(t x) x^{p-1} d t, & \text { if } \quad p \geqq 2 .\end{cases}
$$


It follows that $r^{p}=\psi$, by induction on $p$.

4. The transposes. In order to treat the case in which $\psi$ is not a $p$-th derivative, we introduce $\psi_{k}^{j}(u)$, the $k$-transpose of $\psi^{j}(u)$, defined as follows.

For $0 \leqq j \leqq k \leqq p$ and $u \in \Omega, \psi_{k}^{j}(u)$ is the element of $L_{p+j}(X, Y)$ determined by the relation

$$
\begin{gathered}
C_{k, j} \psi_{k}^{j}(u) x_{1} \ldots x_{p-k+j} h_{1} \ldots h_{k} \\
=\sum_{1 \leqq i_{1}<\ldots<i_{j} \leqq k} \psi^{j}(u) h_{i_{1}} \ldots h_{i_{j}} x_{1} \ldots x_{p-k+j} h_{1} \ldots \hat{h}_{i_{1}} \ldots \hat{h}_{i_{j}} \ldots h_{k},
\end{gathered}
$$

$h_{1}, \ldots, h_{k}, x_{1}, \ldots, x_{p-k+j} \in X$, where the numbers $C_{k, j}$ are binomial coefficients. There are $C_{k, j}$ terms in the summation. In particular,

$$
\psi_{k}^{0}(u)=\psi^{0}(u)=\psi(u), \quad k \leqq p, \quad u \in \Omega,
$$

and

$$
\psi_{k}^{k}(u) x_{1} \ldots x_{p} h_{1} \ldots h_{k}=\psi^{k}(u) h_{1} \ldots h_{k} x_{1} \ldots x_{p}, h_{1}, \ldots, h_{k}, x_{1}, \ldots, x_{p} \in X .
$$

Thus $\psi_{k}^{j}(u)$ exists if $\psi^{j}(u)$ exists. Since $\psi^{j}(u)$ is symmetric in its first $j$ arguments and in its last $p$ arguments, it follows that $\psi_{k}^{j}(u)$ is symmetric in its last $k$ arguments and in its first $p-k+j$ arguments.

Note that

and

$$
\left\|\psi_{k}^{j}(u)\right\| \leqq\left\|\psi^{j}(u)\right\|
$$

$$
\left\|\psi_{k}^{j}(u)-\psi^{j}(u)\right\| \leqq 2\left\|\psi^{j}(u)\right\|, \quad 0 \leqq j \leqq k \leqq p, \quad u \in \Omega .
$$

If the first derivative $\psi^{1}(u)$ is symmetric and if $\psi^{j}(u)$ exists, then

$$
\psi_{k}^{j}(u)=\psi^{j}(u), \quad 0 \leqq j \leqq k \leqq p, \quad u \in \Omega .
$$

This is because $\psi^{j}(u)$ is symmetric, by Proposition 2 .

5. The derivatives of $r$. Suppose that $\psi \in C^{k}$. By Leibnitz' rule,

$$
r^{k}(x)=\int_{0}^{1} \frac{(1-t)^{p-1}}{(p-1) !} D_{x}^{k}\left[\psi(t x) x^{p}\right] d t, \quad x \in \Omega .
$$

In evaluating the interior derivative, we again use differentials. Thus (cf. (9)),

$$
\begin{aligned}
d_{x}^{k}\left[\psi(t x) x^{p}\right] & =\sum_{0 \leqq j \leqq k} \frac{p !}{(p-k+j) !} t^{j} \psi^{j}(t x) \sum_{1 \leqq i_{1}<\ldots<i_{j} \leqq k} h_{i_{1}} \ldots h_{i_{j}} x^{p-k+j} h_{1} \ldots \hat{h}_{i_{1}} \ldots \hat{h}_{i_{j}} \ldots h_{k}, \\
h_{1} & =d_{1} x \in X, \ldots, h_{k}=d_{k} x \in X, \quad x \in \Omega, \quad 0 \leqq t \leqq 1, \quad 1 \leqq k \leqq p .
\end{aligned}
$$

For $j=0$, the inner sum is the solo term $x^{p-k} h_{1} \ldots h_{k}$.

We now introduce the $k$-transposes, in order to arrange that the differentials $h_{1}, \ldots, h_{k}$ occur at the extreme right of each term. By (10),

$$
d_{x}^{k}\left[\psi(t x) x^{p}\right]=\sum_{0 \leqq j \leqq k} \alpha_{k, j} t^{j} \psi_{k}^{j}(t x) x^{p-k+j} h_{1} \ldots h_{k}, \quad x \in \Omega,
$$


where

$$
\alpha_{k, j}=\frac{p ! k !}{(p-k+j) ! j !(k-j) !}, \quad 0 \leqq j \leqq k \leqq p .
$$

Hence

$$
D_{x}^{k}\left[\psi(t x) x^{p}\right]=\sum_{0 \leqq j \leqq k} \alpha_{k, j} t^{j} \psi_{k}^{j}(t x) x^{p-k+j}, \quad 0 \leqq k \leqq p .
$$

Thus, from (13),

$$
r^{k}(x)=\int_{0}^{1} \frac{(1-t)^{p-1}}{(p-1) !} \sum_{0 \leqq j \leqq k} \alpha_{k, j} t^{j} \psi_{k}^{j}(t x) x^{p-k+j} d t, \quad x \in \Omega, \quad k \leqq p,
$$

if $\psi \in C^{k}$.

6. The deviations of $r^{k}$. It is convenient to define $r_{k}$, the pseudo $k$-th derivative of $r$, and $s_{k}$, the deviation of $r^{k}$ from $r_{k}$, as follows:

$$
\left\{\begin{array}{l}
r_{p}=\psi, \\
r_{k}(x)=\int_{0}^{1} \frac{(1-t)^{p-k-1}}{(p-k-1) !} \psi(t x) x^{p-k} d t, \quad x \in \Omega, \quad k<p ; \\
s_{k}(x)=r^{k}(x)-r_{k}(x), \quad x \in \Omega, \quad k \leqq p .
\end{array}\right.
$$

In the one-dimensional case $X=R$, we have $s_{k}(x)=0, x \in \Omega, k \leqq p$. In all cases, $s_{k}(0)=0, k \leqq p$, by Proposition 1 . And

$$
s_{k}(x) h_{1} \ldots h_{k}=0, \quad x \in \Omega, \quad 1 \leqq k \leqq p,
$$

whenever the differentials $h_{1}, \ldots, h_{k}$ are all collinear with $x$, that is, whenever $h_{i}=a_{i} x, a_{i} \in R, i=1, \ldots$, and $k$. As we do not use this fact, we omit its formal proof.

Theorem. If $\psi \in C^{k}$, then for all $x \in \Omega$,

$$
\left\{\begin{array}{l}
s_{0}(x)=0 \\
s_{k}(x)=\int_{0}^{1} \frac{(1-t)^{p-1}}{(p-1) !} \sum_{1 \leqq j \leqq k} \alpha_{k, j} t^{j}\left[\psi_{k}^{j}(t x)-\psi^{j}(t x)\right] x^{p-k+j} d t, \quad 1 \leqq k \leqq p .
\end{array}\right.
$$

Furthermore, one may replace $\psi$ in (19) by $\psi-\varphi$, where $\varphi$ is the p-th derivative of any $C^{p+k}$-map on $\Omega$ to $Y$.

Proof. That $s_{0}=0$ is immediate, by (17) and (4).

Suppose that $k \geqq 1$. We first show that

$$
r_{k}(x)=\int_{0}^{1} \frac{(1-t)^{p-1}}{(p-1) !} \sum_{0 \leqq j \leqq k} \alpha_{k, j} t^{j} \psi^{j}(t x) x^{p-k+j} d t, \quad x \in \Omega, \quad k \leqq p,
$$

an identity similar to (16). 
One may transform the right member by parts, proceeding from $\psi^{k}(t x) x d t=$ $=d_{t} \psi^{k-1}(t x)$ to $\psi(t x)$. The boundary terms will in fact vanish and the integrations terminate in (20). Instead of dealing with the sequence of integrations and the boundary terms, however, one may argue as follows.

The relation (20) holds for all $\psi$ which are $p$-th derivatives, by (5), (16), and (17), since in this case $\psi^{j}(x) \in S_{p+j}(X, Y), x \in \Omega$. It follows that (20) holds for all $\psi$, whether $p$-th derivatives or not, as we now show.

Denote the difference of the two members of (20) by $\delta(x)$. In $\delta(x)$ replace the derivatives $\psi^{j}, j<k$, by their Taylor expansions about the origin with integral remainder in $\psi^{k}$. This, by Fubini's theorem, yields an identity:

$$
\delta(x)=c_{0} \psi(0) x^{p-k}+\ldots+c_{k-1} \psi^{k-1}(0) x^{p-1}+\int_{0}^{1} c(t) \psi^{k}(t x) x^{p} d t, \quad x \in \Omega,
$$

for all $\psi: \Omega \rightarrow S_{p}(X, Y)$, where $c_{i} \in R, i<k$, and $c:[0,1] \rightarrow R$ is continuous.

By suitable choices of $\psi$ which are $p$-th derivatives, we will deduce that $c_{i}=0$, $i<k$, and $c=0$. Thus, take $\psi(x)=a_{i} x^{i}, x \in \Omega$, where $i=0, \ldots$, or $k-1$, and $0 \neq a_{i} \in S_{p+i}(X, Y)$. Since $\psi$ is a $p$-th derivative, (21) reduces to

$$
\delta(x)=0=c_{i} a_{i} x^{p-k+i} i !, \quad x \in \Omega .
$$

Hence $c_{i}=0, i<k$, and the right member of (21) reduces to its integral term.

It remains to show that $c=0$. Take $0 \neq x_{0} \in \Omega$. Put $\widetilde{R}=\operatorname{span} x_{0}$ and $Q=\mathrm{a}$ complement of $\tilde{R}$ in $X$. Thus $X=\tilde{R}+Q$ and $\tilde{R}$ is one-dimensional. Denote the projection of $x$ on $\tilde{R}$ by $x^{\prime}, x \in X$.

Take $0 \neq a \in Y$. Extend the function $c$, by putting $c(u)=c(1), u \geqq 1$, and $c(u)=c(0), u \leqq 0$. Define the map $\theta: \Omega \rightarrow Y$ by the relation

$$
\theta(x)=\theta\left(x^{\prime}\right)=\int_{0}^{1} \frac{(1-t)^{p+k-1}}{(p+k-1) !} c\left(t x^{\prime} / x_{0}\right) x^{p+k} d t a, \quad x \in \Omega .
$$

Here we consider elements of $\tilde{R}$ as scalars. Thus $t x^{\prime} / x_{0}$ is a scalar and $\theta(x)$ is a scalar multiple of $a$, hence an element of $Y$.

Now

$$
\theta^{p+k}(x) h_{1} \ldots h_{p+k}=c\left(x^{\prime} / x_{0}\right) a h_{1}^{\prime} \ldots h_{p+k}^{\prime}, \quad h_{1}, \ldots, h_{p+k} \in X .
$$

This is immediate, by partial differentiation relative to the decomposition $X=\widetilde{R}+Q$. For, from the definition of $\theta$, the pure partials of $\theta$ relative to $\widetilde{R}$ are obtainable from one-dimensional theory (Proposition 1 applied to maps on $\tilde{R}$ ), in particular

$$
D_{\bar{R}}^{p+k} \theta(x)=c\left(x^{\prime} / x_{0}\right) a,
$$

and all other $(p+k)$-th partials vanish.

In (21), put $\psi=\theta^{p}$, a $p$-th derivative. Then $\delta(x)=0, x \in \Omega$. Put $x=x_{0} \in \tilde{R}$. Then $x^{\prime}=x_{0}^{\prime}=x_{0}$,

$$
\theta^{p+k}\left(t x_{0}\right) x_{0}^{p} h_{1} \ldots h_{k}=\psi^{k}\left(t x_{0}\right) x_{0}^{p} h_{1} \ldots h_{k}=c(t) a x_{0}^{p} h_{1}^{\prime} \ldots h_{k}^{\prime} ; \quad h_{1}, \ldots, h_{k} \in X,
$$


and

Hence

$$
\delta\left(x_{0}\right) h_{1} \ldots h_{k}=0=\int_{0}^{1} c(t) c(t) a x_{0}^{p} h_{1}^{\prime} \ldots h_{k}^{\prime} d t
$$

$$
\int_{0}^{1}[c(t)]^{2} d t=0
$$

and $c=0$. Thus (20) is established.

Now (18) follows from (16) and (20), since the term for $j=0$, eliminated from the summation in (19), vanishes.

That one may replace $\psi$ by $\psi-\varphi$, where $\varphi$ is a $p$-th derivative, follows from the fact that $\varphi_{k}^{j}=\varphi^{j}, 0 \leqq j \leqq k$, since $\varphi^{j}$ is a $(p+j)$-th derivative and therefore symmetric.

For $p=1$, the relation (18) agrees with the formula for $\operatorname{rot} \varphi A$ in [3, p. 144].

7. Comparison of $f$ and $g$. In this section, we assume that $\psi, \varphi \in C^{j}$ whenever $\psi^{j}, \varphi^{j}$ appear in a statement.

By (1) and (3),

$$
\begin{gathered}
g^{k}(x)-f^{k}(x)=b_{k}-a_{k}+\ldots+\frac{b_{p-1}-a_{p-1}}{(p-k-1) !} x^{p-k-1}+r^{k}(x) \\
-\int_{0}^{1} \frac{(1-t)^{p-k-1}}{(p-k-1) !} \varphi(t x) x^{p-k} d t, \quad x \in \Omega, \quad k<p .
\end{gathered}
$$

Hence, by (18), (17), and (2),

$$
\begin{gathered}
g^{k}(x)-f^{k}(x)=b_{k}-a_{k}+\ldots+\frac{b_{p-1}-a_{p-1}}{(p-k-1) !} x^{p-k-1} \\
+\int_{0}^{1} \frac{(1-t)^{p-k-1}}{(p-k-1) !}[\psi(t x)-\varphi(t x)] x^{p-k} d t+s_{k}(x), \quad x \in \Omega, \quad k<p,
\end{gathered}
$$

and

$$
g^{p}(x)-f^{p}(x)=\psi(x)-\varphi(x)+s_{p}(x), \quad x \in \Omega .
$$

These differences are readily appraised. In the first place, writing $\|z, x\|$ for $\sup _{t \in[0,1]}\|z(t x)\|$, we have

$$
\begin{gathered}
\text { (*) } \quad\left\|s_{k}(x)\right\| \leqq \sum_{1 \leqq j \leqq k} \frac{2 p ! k !}{(p+j) !(p-k+j) !(k-j) !}\left\|\left(\psi^{j}-\varphi^{j}\right), x\right\|\|x\|^{p-k+j}, \\
x \in \Omega, \quad 1 \leqq k \leqq p .
\end{gathered}
$$

This inequality follows from (19) with $\psi$ replaced by $\psi-\varphi$, and from (12), (15), and the fact that

$$
\int_{0}^{1} \frac{(1-t)^{p-1}}{(p-1) !} t^{j} d t=\frac{j !}{(p+j) !} .
$$


And, by (22), (23),

(*)

$$
\begin{gathered}
\left\|g^{k}(x)-f^{k}(x)\right\| \leqq\left\|b_{k}-a_{k}\right\|+\ldots+\frac{\left\|b_{p-1}-a_{p-1}\right\|}{(p-k-1) !}\|x\|^{p-k-1} \\
+\frac{\|(\psi-\varphi), x\|}{(p-k) !}\|x\|^{p-k}+\left\|s_{k}(x)\right\|, \quad x \in \Omega, \quad k<p
\end{gathered}
$$

and

(*)

$$
\left\|g^{p}(x)-f^{p}(x)\right\| \leqq\|\psi(x)-\varphi(x)\|+\left\|s_{p}(x)\right\|, \quad x \in \Omega .
$$

Added in proof. The results of the present paper hold for complex as well as real Banach spaces $X$ and $Y$.

\section{References}

[1] Cartan, Henri: Calcul différentiel. - Hermann, Paris, 1967.

[2] LANG, Serge: Introduction to differentiable manifolds. - John Wiley and Sons, New York, 1962.

[3] Nevanlinna, F. and R.: Absolute Analysis. — Springer-Verlag, Berlin-Göttingen-Heidelberg, 1959.

The City University of New York

Queens College

Flushing, New York 11367

USA

Current address:

ob dem Huegliacker 16

4102 Binningen

Switzerland

Received 13 June 1977 